\title{
INDEX OF VOLUME 47
}

\section{INDEX OF ABSTRACTS, NOTES AND OTHER ITEMS}

Abstracts of Papers Presented to the Society:

Subject:

Algebra and Theory of Numbers, 30, 50, 201, 217, 373, 553, 683, 856.

Analysis, 33, 203, 381, 555, 691, 860.

Applied Mathematics, 44, 209, 390, 559, 703, 863.

Geometry, 46, 210, 392, 560, 705, 863.

Logic and Foundations, 215, 708.

Statistics and Probability, 49, 216, 394, 561, 709, 864.

Topology, 52, 217, 397, 561, 711, 866.

Authors:*

Agnew, R. P., 187 (203), 530 (555), 841 (691), 842 (692), 844 (692); Albert, A. A., 842 (683), 844 (683); Albert, G. E., 521 (397), 522 (397); Allen, E. F., 840 (705); Allen, E. S., 187 (209); Ambrose, W., 186 (33), 842 (697); Arnold, H. A., 529 (561), 530 (562); Arnold, K. J., 839 (709); Atanasoff, J. V., 529 (392).

Baer, R., 521 (373), 522 (392), 845 (683); Baker, G. A., 174 (49), 174 (49); Bal, Y. K., 528 (392); Bankier, J. D., 839 (692); Basch, A., 529 (561); Bateman, H., 174 (44); Beckenbach, E. F., 340 (203), 520 (381), 845 (692), (705), (862); Bell, E. T., 174 (50); Bell, J. H., 521 (201); Bell, P. O., 184 (46); Bergman, S., 340 (204), 340 (210), 528 (381), 530 (555), 843 (692); Bers, L., 339 (204), 530 (381), (860); Best, E., 844 (688); Biot, M. A., 174 (44); Birkhoff, G., 844 (708); Bjerknes, J., 174 (44); Blake, A., 340 (216); Blumberg, H., 840 (708); Blumenthal, L. M., 184 (47), 185 (47), 340 (210); Boas, R. P., 185 (34), 339 (204), 841 (556); Botts, T. A., 843 (562); Bourgin, D. G., 528 (556), 840 (693), (711); Brauer, A. T., 4 (46; 891), 841 (690); Brauer, R., 521 (373), 842 (684), 845 (684); Brown, A. B., 4 (46; 891), (860); Bruck, R. H., 184 (30), 528 (373), 528 (374), 844 (684), 844 (684), (856); Buell, E. L., 844 (703); Burcham, P. B., 521 (382); Burr, I. W., 839 (709).

Cairns, S. S., (711), (711); Cameron, R. H., 840 (693); Camp, C. C., 184 (30), 844 (693); Campaigne, H. H., 840 (685); Campbell, A. D., 842 (685), (856); Carlitz, L., 187 (50), 530 (380), 530 (380), 530 (554), 845 (690), 845 (690); Carruth, P. W., 843 (685); Cassity, C. R., 184 (47); Clausner, F. H., 174 (210); Clifford, A. H., 341 (201), 341 (201), 530 (553); Coburn, N., 186 (47), 186 (47), 517 (393), 843 (705); Coleman, J. B., 839 (710); Comentez, G., (863); Copeland, A. H., 530 (394); Courant, R., 187 (48), 529 (382); Court, N. A., 184 (48), 186 (48); Cowan, R., 184 (34); Crow, E. L., 520 (382); Curtiss, J. H., 185 (34), 186 (34), 841 (556), (710).

Daly, J. F., 839 (864); Dantzig, G. B., 517 (395); Davids, N., (704); Day, M. M., 6 (46; 748), 6 (46; 891), 840 (694), 844 (693), 844 (693); DeCicco, J. J., 187 (214), 340 (213), 341 (211), 341 (212), 341 (213), 521 (211), 522 (211), 522 (213), 530 (210), 839 (706), 843 (393), 843 (560), 843 (560), 843 (560), 843 (707), (863); Dehn, M., 841 (711); Derry, D., 184 (51); Diehl, H., 187 (209); Dines, L. L., 184 (35); Dobbie, J. M., 841 (690); Douglas, J., 186 (35); Dribin, D. M., 522 (374); Duffin, R. J., 521 (383), 839 (694), 840 (685), 841 (694); Dushnik, B., 6 (52); Duthie, W. D., 185 (30).

* Numbers within parentheses refer to the page numbers for abstracts, and those outside parentheses refer to the pages for titles as listed in reports of meetings. 
Eachus, J. J., 185 (35), 841 (694); Earl, J. M., 841 (695); Eilenberg, S., 6 (35), 185 (52), 187 (53), 521 (397), 529 (398), 530 (398), 841 (866); Epstein, B., 521 (383); Erdös, P., 341 (217), 845 (695); Everett, C. J., 186 (31), 843 (553).

Feld, J. M., 340 (212); Feller, W. K., 842 (710); Fialkow, A., 4 (46; 891), 340 (212); Ficken, F. A., 528 (562), 840 (712); Folley, K. W., 6 (36); Ford, L. R., 839 (695); Forsythe, G. E., 4 (46; 892), 530 (395); Fort, T., (857); Foster, M. C., 341 (212); Fox, R. H., 841 (712); Friedman, B., 841 (695); Friedrichs, K. O., 529 (559); Frink, O., 844 (712).

Garabedian, H. L., 185 (36), 341 (205), 844 (696), 844 (696); Gatewood, B. E., 184 (45); Geiringer, H. P., 842 (864); Gelbart, A., 339 (205), 845 (696); Giese, J. H., 840 (706); Glass, T. F., 842 (696); Goldstine, H. H., 341 (205), 520 (383); Gorn, S., 4 (46; 892); Gravalos, F. G., 529 (559); Green, J. W., 183 (36); Greenwood, J. A., 529 (395); Greenwood, R. E., 185 (36); Griffiths, L. W., 521 (380); Grove, V. G., 521 (393); Gumbel, E. J., (865).

Hall, M., 340 (201); Hall, N. A., 4 (46; 892); Halmos, P. R., 339 (206), 842 (696); Hamilton, H. J., 173 (36); Hammer, P. C., 522 (396); Harrington, W. J., 528 (383); Harrold, O. G., 185 (53), 841 (712); Hay, G. E., 520 (390); Hedge, L. B., 183 (37); Hedlund, G. A., 843 (707); Heins, A. E., 186 (37), 520 (390); Heins, M. H., 528 (556); Herzog, F., 841 (686); Hestenes, M. R., 187 (37); Heyda, J. F., 520 (384); Hildebrand, F. B., 187 (45); Hille, E., 4 (37), 530 (557); Hohn, F. E., 839 (706); Hollcroft, T. R., 839 (707); Horenstein, W., (861); Householder, A. S., 520 (391), 842 (703); Hsu, C. T., 530 (396); Hurewicz, W., 529 (562); Hyers, D. H., 520 (206), 845 (708).

Jackson, D., 521 (384); James, R. D., 841 (691); Jennings, S. A., 844 (686); John, F., 185 (38); Jones, A. W., 843 (553); Jones, B. W., 843 (686); Jones, F. B., 6 (53), 185 (53), 341 (217), 840 (697).

Kac, M., 529 (554), 842 (692), 844 (697); Kakutani, S., 340 (218), 842 (697), 845 (697); Kalisch, G. K., 842 (686); Kaplan, W., 842 (704); Kaplansky, I., 340 (31); von Kármán, T., 174 (45); Kasner, E., 187 (214), 340 (213), 341 (212), 341 (213), 522 (213), 843 (393), 843 (560), 843 (560), 843 (707); Kelley, J. L., 184 (218); Kershner, R. B., 842 (698); Kiokemeister, F., 522 (374); Knobelauch, E. A., 844 (713); Kober, H., 341 (384); Kolchin, E. R., 340 (202); Komm, H., 840 (713); Korn, A., (863).

Laden, H. N., 339 (206); Lane, R. E., 522 (385); Langer, R. E., 840 (698); LaPaz, L., 522 (385); LaSalle, J. P., 174 (38), 174 (38), 183 (53), 843 (713); Lehmer, D. H., 174 (51), 517 (380), (857); Lehner, J., 341 (217), 528 (554); Leighton, W., 186 (39), 839 (557), 839 (692), 842 (696); Levi, H., 340 (202), (857); Levinson, A., (704); Levit, R. J., (857); Lewy, H., 518 (385); Lonseth, A. T., 520 (385); Loomis, L. H., 4 (46; 893), 530 (386), 530 (386); Lorch, E. R., 4 (46; 604), 528 (386), (860); Lowan, A. N., (704), (861); Lubben, R. G., 840 (714).

McClay, D. T., 528 (560); McEwen, G. F., 173 (49); McMillan, B., 845 (861); McShane, E. J., 528 (557); MacDuffee, C. C., 529 (374); Mackey, G. W., (861); MacLane, S., 341 (201), 530 (375), 530 (375), 530 (375), 841 (866); Madow, W. G., 340 (216); Maharam, D., 339 (206); Maker, P. T., 4 (46; 894); Mancill, J. D., 843 (558), 844 (698); Mandelbrojt, S., 184 (207), 842 (699); Mansfield, R., 843 (699); Marcinkiewicz, J., 185 (39); Marden, M., 840 (687); Martin, R. S., 4 (39); Mersman, W., 517 (391); Meyer, H., 840 (699); Milgram, A. N., 6 (54), 184 (54); Miller, E. W., 6 (52); Miller, H. C., 185 (54), 186 (55), 187 (55), 522 (398); Mittleman, D., 341 (214); Montgomery, D., 844 (687); Mood, A. M., 
839 (710); Moore, C. N., 185 (39); Moore, M. G., 845 (699); Moore, R. L., 841 (866); Morse, M., 843 (707); Murdoch, D. C., 840 (687); Myers, S. B., 184 (49), $187(55)$.

von Neumann, J., 842 (696); Neyman, J., 174 (50); Nichols, G. D., 186 (39); Nicholson, E. H., 845 (700); Nielsen, K. L., 528 (386); Nilson, E. N., 183 (43); Niven, I. M., 521 (375), 529 (375), 845 (687), 845 (691).

Oberg, E. N., 843 (558); Oboukhoff, N. M., 187 (207); O'Connor, R. E., (858), (859), (859); Odle, J. W., 6 (55); Oldenburger, R., 184 (31), 840 (688); Olds, C. D., 522 (381), 522 (387); Opatowski, I., 842 (704); Ore, O., 340 (202), 341 (202); Ott, E. R., 184 (46; 894).

Pall, G., 187 (51), 528 (217), 841 (859), 843 (688); Pate, R. S., 521 (376), 840 (685); Paydon, J. F., 839 (862); Peebles, G. H., 520 (387); Perez, F., 4 (46; 894); Perlin, I. E., 183 (40), 840 (700); Perlis, S., 522 (376); Phillips, R. S., 840 (700); Pitcher, E., 528 (376); Pollard, H., (700); Pólya, G., 339 (207), 529 (558); Prenowitz, W., (864); Puckett, W. T., 517 (398).

Rademacher, H. A., 341 (217); Rad6, T., 520 (207), 529 (387); Rainich, G. Y., 6 (32); Randels, W. C., 520 (387); Rannie, W. D., 174 (46); Reade, M., (862); Reid, W. T., 522 (558); Reissner, E., 186 (46), 842 (704); Riblet, H. J., 530 (376); Rickart, C. E., 520 (387); Ritt, J. F., (862); Robertson, M. S., 530 (558); Robinson, C. V., 184 (47), (864); Robinson, L. B., 4 (46; 894), 4 (46; 895), 530 (559); Robinson, N., 184 (214), 839 (561); Robinson, R. M., 174 (51); Rosenbloom, P. C., 340 (203); Rosenthal, A., 842 (701); Rosenthal, J. E., 4 (46; 895); Rosser, B., 184 (51), 528 (383), 529 (554); Rutt, N. E., 183 (32), 186 (56).

Salem, R., 341 (207), 341 (208), 528 (388), 531 (388), 842 (701); Sard, A., 186 (40); Savage, L. J., 521 (394); Schaeffer, A. C., 839 (694); Schatten, R., (862); Scheffé, H., 341 (216), 517 (296), 522 (388); Scherk, P., 4 (46; 895), 340 (215); Schilling, O. F. G., 530 (375), 530 (375), 530 (375); Schoenberg, I. J., 339 (389), 341 (208); Schwartz, A., 839 (708); Schwartz, H. M., 186 (40), 186 (40), 518 (389); Schweigert, G. E., 185 (56), 522 (399); Schweitzer, A. R., (709); Scott, W. M., 528 (376); Scott, W. R., 520 (389); Scott, W. T., 6 (46; 895), 6 (46; 896), 521 (208), 521 (389); Sears, W. R., 174 (45); Segal, I. E., 4 (46; 896); Serbin, H., 185 (41); Shanks, M. E., 521 (399); Shohat, J. A., 341 (209), 529 (390); Simmons, H. A., 6 (51), 521 (377), 522 (377), 843 (688); Smiley, M. F., 186 (32), 341 (377), 528 (376); Snapper, E., 340 (203), (858); Snyder, W. S., 183 (41); Sokolnikoff, I. S., 520 (391); Sorgenfrey, R. H., 184 (56); Spencer, D. C., 843 (692), 844 (701); Spitzbart, A., 187 (41); Steenrod, N. E., 522 (399), 841 (866); Stephens, R. C., 341 (218); Stewart, B. M., 521 (378); Stewart, H. J., 174 (46); Sugar, A., 341 (215); Sutton, R. M., 529 (392); Swain, R. L., 184 (56), 187 (55), 187 (56); Swinford, L. H., 517 (390); Swingle, P. M., 174 (57), 185 (57); Szász, O., 185 (41), 521 (390), 839 (701); Szegö, G., 845 (695).

Taussky, O., 844 (688); Taylor, A. E., 174 (42); Thielman, H. P., 520 (378); Thomas, J. M., 184 (42); Thorne, C. J., 529 (392); Thrall, R. M., 528 (378), 842 (689); Thron, W. J., 839 (557); Trjitzinsky, W. J., 187 (42), 528 (559); Tucker, A. W., 6 (57), 517 (400), 522 (399), 841 (714).

Ulam, S. M., 840 (702), 845 (702), 845 (708).

Vandiver, H. S., 845 (859); Vickery, C. W., 185 (57).

Wade, L. I., 184 (52); Wade, T. L., 184 (32), 528 (373), 528 (374), 844 (684), 844 (689), (856); Wagner, R. W., 183 (33); Wahlin, G. E., 340 (210); Wakerling, R. K., 518 (394); Wald, A., 340 (396), 839 (865); Walker, G. L., 529 (379); Wall, H. S., 4 (46; 760), 6 (46; 895), 6 (46; 896), 185 (42), 521 (208), 521 (389), 
(863); Wallace, A. D., 529 (400), 843 (714); Wallman, H., 841 (866); Walsh, J. L., 183 (43); Ward, M., 174 (33), 517 (379); Warschawski, S. E., 185 (209); Weinstein, A., 340 (390), 529 (392), 843 (705); Weisner, L., 531 (379), 844 (689); Wernick, W., 4 (46; 896), 5 (46; 896); Weyl, H., 186 (33); White, P. A., 529 (400); Whitehead, G. W., 522 (400); Whiteman, A. L., 841 (555); Whitmore, W.F., 174 (43); Whitney, H., 5 (46; 897), 340 (218); Whyburn, G. T., 342 (219), 529 (401), 531 (401), 531 (401); Widder, D. V., 185 (34), 186 (43); Wiener, N., 529 (558); Wilcox, L. R., 520 (379), 842 (689), 845 (858); Wilder, R. L., 185 (58), 186 (58), 186 (58); Wolf, F., 840 (702), 844 (702); Wolfowitz, J., 340 (216); Woodbury, M. A., 520 (379); Woolson, J. R., 174 (44); Wundheiler, A., 342 (215).

Young, G. S., 841 (715), 845 (715); Youngs, J. W. T., 521 (397), 842 (703), (715).

Zariski, O., 187 (215); Zippin, L., 844 (687); Zorn, M., 174 (52), 518 (381); Zygmund, A., 185 (39).

Academies, Associations, Congresses, and Societies:

American Mathematical Society: Annual Meeting (1940), 175; Annual Meeting (1941), 179; Auditors' Report, 181; Budget, 181; Bulletin, 24, 527; By-Laws, 182, 334, 838; Colloquium Lectures, 179, 526, 834; Colloquium, 527; Committee on Addresses in Applied Mathematics, 183; on Circulation for Mathematical Reviews, 838; on Places of Meetings, 526; on Dues for Persons Drafted or Enlisted for Military Service, 838; on Introduction of New Series of Mathematical Books, 183, 527; 838; on Invited Addresses, 179, 366; on Nominations, 182, 526; on Publicity, 179; on War Preparedness, 182, 829, 836, 837, 850; Council, Voting Representatives, 527; Dates and Places of Meetings, 181, 526, 837; Election of Officers, 179, 527; Gibbs Lectures, 834, 837; Librarian's Report, 182; Mathematical Reviews, 1, 182, 838; Mathematical Surveys, 838; New Members Admitted, 177, 524, 836; Representatives on the Council of American Association for the Advancement of Science, 838; at Inauguration of President of Beaver College, 179; at Fiftieth Anniversary Celebration of University of Chicago, 526; at Inauguration of President of Drury College, 179; on Duke Mathematical Journal, 179; at Centenary Celebration of Fordham University, 526; at Inauguration of Sixth President of Grinnell College, 179; at Inauguration of President of The State University of Iowa, 526; on National Research Council, 838; at Inauguration of President of Northeastern University, 179; at One Hundred and Seventyfifth Anniversary of the Founding of Rutgers University, 837; at Inauguration of Fourth President of Wheaton College, 526; Statistics, 179, 182; Summer Meeting (1941), 832; (1942), 526; Transactions, 24, 527; Treasurer's Report, 181.

American Association for the Advancement of Science, 25, 197, 679, 838; American Institute of Electrical Engineers, 850; Carnegie Corporation, 548; Committee on Scientific Aids to Learning, 1, 182; Econometric Society, 832; English Physical Society, 850; Geological Society of America, 25; Institute of Mathematical Statistics, 832; International Congress for the Unity of Science, 366; Jablonowskischen Gesellschaft der Wissenschaften, 851; London Mathematical Society, 679; Mathematical Association of America, 25, 175, 366, 548, 829, 832; National Council of Teachers of Mathematics, 175, 197; National Research Council, 1, 25, 838; Royal Society, 198.

Books, Announcement of New, 25, 59, 197, 220, 366, 403, 564, 716, 838, 868.

Doctorates, American, 367. 
Journals:

American Journal of Mathematics, 182, 527; Bulletin of the American Mathematical Society, 24, 527; Duke Mathematical Journal, 179; Mathematical Reviews, 1, 182, 838; Transactions of the American Geophysical Union, 25; Transactions of the American Mathematical Society, 24, 527.

National Research Fellowships, 680.

\section{Personal Notes:}

Adams, C. R., 179, 366, 524, 835; Adler, F., 851; Agnew, R. P., 24, 526; Alaoglu, L., 24; Albert, A. A., 182, 367, 519, 550, 834; Alt, F., 366; Ambrose, W., 682; Anderson, P. H., 367; Archibald, R. G., 179, 526; Arens, R. F., 549, 680; Arnold, H. A., 682; Aroian, L. A., 367; Artin, E., 834; Askovitz, S. I., 548, 549; Assadourian, F., 367; Aucoin, A. A., 367; Ayres, W. L., 24, 179, 550, 837.

Baer, R., 24; Bargmann, V., 851; Barnard, R. W., 24; Barnett, I. A., 24; Barron, J. J., 550; Bartel, K., 854; Bartky, W., 24; Basoco, M. A., 550; Basye, R. E., 28; Bateman, H., 173, 182, 837; Baumberger, J. R. R., 549; Beatty, R. E., 549; Beaumont, R. A., 367; Beckenbach, E. F., 5, 519; Begle, E. G., 367, 680; Bell, E. T., 197, 837; Bell, J. H., 680; Bell, P. O., 24, 680; Bellman, R., 548, 549; Benac, T. J., 854; Bennett, A. A., 24, 835; Bennett, W. R., 850; Berry, A. C., 680; Betz, E. E., 854; Bewley, L. V., 837; Birkhoff, G., 24, 679, 834; Birkhoff, G. D., 24, 679, 851; Blackwell, D., 851; Blanche, E. E., 854; Blichfeldt, H. F., 197; Bliss, G. A., 182, 366, 519, 526, 549, 834, 836; Bloom, M., 28; Blumberg, H., 24; Blumberg, J. O., 367; Blumenthal, L. M., 519, 837; Boas, R. P., 24; Bobonis, A., 680; Bochner, S., 24, 834, 835; Bohnenblust, H. F., 339; Bohnert, J. I:, 367; Bolwell, R. W., 524; Bolza, O., 835; Boyer, C. B., 550; Bradley, A. D., 198; Brahana, H. R., 24; Branson, H. R., 852; Brauer, A. T., 24, 851; Brauer, R., 24, 550, 834, 851; Bridgman, P. W., 679; Brill, J., 855; Brink, R. W., 367, 834; Brinkmann, H. W., 24; Brown, A. A. F., 198, 367; Brown, A. B., 24; Brown, B. H., 367; Brown, B. L., 550; Brown, G. W., 198, 367; Brown, O. E., 550; Bruck, R. H., 367; Buchanan, H. E., 24, 177; Buell, E. L., 682; Bullen, K. E., 26; Burington, R. S., 182; Burns, H. E., 200; Burr, I. W., 852; Bush, V., 679; Bushey, Jewell H., 334; Butts, W. H., 855; Byrne, R., 200.

Cairns, S. S., 3; Cairns, W. D., 526, 548, 850; Calabi, E., 549; Campbell, A. D., 526; Canaday, E. F., 368; Carroll, I. S., 550; Carson, J. R., 29; Carver, W. B., 526; Caton, W. B., 26, 852; Chandrasekhar, S., 851; Chapman, S., 548, 850; Chauvenet, W., 197; Chevalley, C., 26; Chiarulli, P., 548; Chittenden, E. W., 24, 179, 181, 183, 519, 527; Church, A., 24; Churchill, R. V., 24, 519, 854; Clark, C. E., 680; Clarkson, J. A., 24; Clement, M. D., 854; Clifford, A. H., 24, 550; Clippinger, R. F., 368; Coble, A. B., 24, 524, 526, 837; Coddington, E. A., 551; Cohen, A., 198; Cohen, A. C., 28; Cohen, L. W., 176, 548; Cohn, H., 549; Cole, K. S., 851; Cole, R. H., 368; Coleman, W. B., 854; Comenetz, G., 24; Cook, R. H., 368; Coolidge, J. L., 182; Copeland, A. H., 24; Cosby, B., 682; Coulson, C. A., 549; Courant, R., 183, 524; Cox, G. M., 198; Cox, W. E., 368; Coxeter, H. S. M., 24; Craig, A. T., 835; Cramlet, C. M., 24; Crawford, L., 366; Cronvich, L. L., 682; Crow, E. L., 682; Cunningham, A. B., 368; Curry, H. B., 3, 182; Curtiss, D. R., 526, 835, 836.

Daly, J. F., 371; Dancoff, S. M., 851; van Dantzig, D., 851; Davenport, H., 679; Davids, N., 368, 851; Davis, H. T., 366; Day, M. M., 24, 200; Dean, J., 366; DeCicco, J. J., 24; Den Hartog, J. P., 524; Dickson, L. E., 679, 834; Dil- 
worth, R. P., 24, 854; Dimsdale, B., 368; Dines, L. L., 519; Dobbie, J. M., 28; Doob, J. L., 24, 851; Dorsett, R. D., 198; Douglas, J., 851; Dresden, A., 835, 836; Dressel, F. G., 852; Dressel, P. L., 26; Duffin, R. J., 28; Dunford, N., 24, 527, 834; Dunlap, L. T., 198; Durand, W. F., 852; Durfee, W. H., 372; Dushnik, B., 24; Duthie, W. D., 368; Duval, E. P. R., 26; Dyer-Bennet, J., 368.

Eaton, J. E., 372; Eberhart, P., 680; Edwards, O. C., 855; Eilenberg, S., 852; Eisenhart, L. P., 548, 838; Engstrom, H. T., 182; Erkiletian, D. H., 551; Esterman, T., 550; Ettlinger, H. J., 176; Evans, G. C., 173, 176, 177, 178, 182, 517, 549, 837; Everett, C. J., 368, 682; Ewing, G. M., 28.

Fan, S.-C., 368; Farrer, H. E., 850; Favila, R. A., 28; Feller, W. K., 24, 680; Finan, E. J., 179, 198; Fischer, C. H., 854; Fisher, R. A., 198; Fleddermann, H. T., 368; Flores, C., 851; Fobes, M. P., 200; Foote, J. R., 200; Ford, L. R., 24, 180; Forsythe, A. I., 682; Forsythe, G. E., 682; Fort, T., 179, 527; Foster, R. M., 334; Franklin, P., 182; Friedrichs, K. O., 334; Frink, O., 24; Frisch, E., 368; Fry, T. C., 3, 176, 182, 837; Fryer, H. C., 198, 368; Fubini, G., 24, 851; Fuller, G., 198.

Gaines, R. E., 852; Galbraith, A. S., 852; Garabedian, H. L., 24; Garrison, G. N., 371; Gaskell, R. E., 368; Gelbart, A. M., 368; Gentry, F. C., 852; Gergen, J. J., 24; Giese, J. H., 200, 368, 854; Gilbert, P. W., 368; Gill, B. P., 180; Gillam, B. E., 368; Givens, J. W., 551; Gleason, A. M., 549; Glover, J. W., 855; Gödel, K., 851; Godfrey, E. L., 854; Goheen, H. E., 28, 369; Goldsworthy, E. C., 182; Golomb, M., 28; Gordon, W. O., 26; Gould, S. H., 550; Grable, E. S., 682; Graustein, W. C., 200, 527; Graves, L. M., 180, 366, 519, 526, 835, 838, 851; Green, J. W., 680; Greer, E., 200; Griffin, F. L., 182, 836; Griffin, H. M., 550; Guenther, P. E., 682; Gunderson, N. G., 200.

Hadlock, E. H., 551, 680; Hagen, B. L., 198; Hall, D. W., 24; Hall, M., 680; Hallett, W. N., 852; Halmos, P. R., 851; Hamilton, H. J., 680; Hammer, P. C., 200; Hansman, M. M., 854; Harding, A. M., 852; Hardy, G. H., 198; Harrington, W. J., 682; Hart, W. L., 24, 182, 850; Hawkes, H. E., 679; Hayes, E. H., 200; Hayes, G. M., 552; Hazard, K. E., 28, 369, 682; Hazeltine, A., 850; H'Doubler, F. T., 179; Hebert, P. M., 176; Hebert, Mrs. P. M., 176; Hedlund, G. A., 24; Hedrick, E. R., 173; Hedrick, Mrs. E. R., 173; Heins, M. H., 369, 851; Henderson, R., 527; Hendrickson, M., 28; Herriot, J. G., 552; Hestenes, A. D., 369; Hestenes, M. R., 24, 519; Heyda, J. F., 369; Highberg, I. E., 680; Hildebrand, F. B., 369; Hildebrandt, T. H., 182, 183, 527, 834, 835; Hille, E., 3, 24, 25, 179, 180, 197, 366, 367, 527; Hinrichsen, J. J. L., 198; Hochschild, G. P., 851; Hodge, F. H., 199; Hoheisel, G., 26; Hollcroft, T. R., 179, 180, 366, 526; Hostetter, I. M., 852; Hotelling, H., 334, 366; Huff, W. N., 28, 551; Humphreys, M. G., 680; Huntington, E. V., 179, 197, 549; Hurewicz, W., 24; Hurwitz, W. A., 182; Huskey, H. D., 854; Hutchinson, L. C., 369, 854; Hutchinson, L. P., 199.

Ince, E. L., 552; Ingraham, M. H., 179, 182, 366, 835, 838.

Jackson, D., 182, 835; Jackson, R. F., 369; Jacobson, N., 24, 26, 176, 834; James, H. M., 524; James, R. D., 24; Jeffery, R. L., 24, 834; Jenkins, E. D., 681; Jenks, F. P., 369; Jenness, J. R., 27; Jennings, S. A., 27; Johanson, R. N., 852; Johnson, D. F., 200; Johnston, F. E., 179; Jones, B. W., 24; Jones, H. J., 200; Jordan, H. A., 27; Joseph, J. A., 854.

Kac, M., 24; Kakutani, S., 851; Kalisch, G. K., 851; Kamel, H., 548; van Kampen, E. R., 24; Kaplan, E. L., 549; Kaplansky, I., 682; von Kármán, T., 173; Kasner, E., 24; Kelley, J. L., 369; Kendig, P. M., 852; Key, F. B., 854; Kibbey, D. E., 551; Kieval, H. S., 854; Kiokemeister, F., 28, 369; Kirchen, C. J., 
854; Klamkin, M., 549; Kleene, S. C., 24, 681; Kline, J. R., 3, 24, 176, 179, 180, 182, 366, 549, 850; Knapp, G. A., 200; Knight, L. C., 27; Knobelauch, E. A., 28; Koehler, F., 24, 199; Kolchin, E. R., 680; Koopman, B. O., 182; Kullback, S., 182.

Laden, H. N., 854; Ladue, P., 855; Lane, E. P., 24; Lange, O., 366; Langer, R. E., 182, 367; Langhaar, H. L., 369; LaSalle, J. P., 854; Lasley, J. W., 679; Latimer, C. G., 24, 834; Lawwill, S. J., 854; Lax, B., 549; Le Caine, J. S., 682; Le Corbeiller, P., 850; Lee, T. H., 27; Lefschetz, S., 834, 835; Lehmer, D. H., 24; Lehner, J., 854; Leib, D. D., 682; Leibler, R. A., 28; Levin, J. H., 369; Levinson, N., 837; Lewis, D. C., 24, 551, 679; Lewis, P. E., 369; Lewy, H., 182; Lindquist, C. B., 682; Longley, W. R., 180; Loomis, E. S., 372; Loomis, L. H., 551; Lorch, E. R., 24, 179; Lösch, F., 26; Loud, W. S., 549; Lovett, E. O., 852; Lowan, A. N., 27; Luckey, R. R. R., 200.

McClay, D. T., 28, 369; McClenon, R. B., 526; McCoy, N. H., 24, 834; McGaughey, A. W., 369, 682; McKean, R. A., 681; McKinsey, J. C. C., 24; McMaster, A. S., 854; McShane, E. J., 24, 179, 182, 526, 527, 835; MacColl, L. A., 850; MacDougal, H. B., 681; MacDuffee, C. C., 524, 527; Mackey, G. W., 851; MacLane, G. R., 549; MacLane, S., 24, 25, 176, 372, 551, 835; Macphail, M. S., 854; Macrosty, H. W., 372; Maddaus, I., 369, 854; Magnusson, C. E., 855; Maharam, D., 369, 851; Mandelbrojt, S., 199, 681; Maninng, R., 854; Manson, S., 549; Many, A. E., 681; Maple, C. G., 854; March, H. W., 24; Marden, M., 24, 27; Marquis, R. H., 681; Martin, A. V., 369; Martin, W. T., 27; Mathisson, M., 200; Mears, F. M., 179; Meder, A. E., 179, 526; Menger, K., 24, 366; Merrill, D. M., 549; Merriman, G. M., 24; Mewborn, A. B., 369; Meyer, H. L., 854; Michal, A. D., 197; Michel, R. J., 27, 681; Mickle, E. J., 682; Miller, C. E., 369; Miller, D. C., 372; Miller, E. W., 853; von Mises, R. E., 24; Mitchell, W. L., 28, 369; Montgomery, D., 24, 679, 851; Montgomery, J. C., 370; Mood, A. M., 200, 370; Moody, E. I., 552; Moore, G. E., 199; Moore, M. G., 28; Moran, C. W., 370; Mordell, L. J., 679; Morehead, J. C., 27; Morgan, W. B., 854; Morkovin, V., 854; Morrey, C. B., 24; Morris, W. L., 370; Morrow, R. C., 28; Morse, M., 177, 179, 180, 182, 197, 334, 366, 524, 526, 834, 835, 837; Morse, P. M., 524; Moulton, E. J., 182; Moursund, A. F., 24; Muhly, H. T., 28, 370; Mullins, G. W., 180; Mumford, C. G., 370; Muncelle, M., 177; Munroe, F. L., 681; Murnaghan, F. D., 182, 523; Murray, F. J., 179, 523; Myers, S. B., 24, 176.

Nash, J. P., 370; Nelson, A. L., 5; Nesbitt, C. J., 853; Neuhaus, A., 370; von Neumann, J., 182, 834, 835, 838; Newell, H. E., 370; Newsom, C. V., 177; Neyman, J., 197; Nielsen, K. L., 370; Nilson, E. N., 682.

Odle, J. W., 28; Ollmann, L. F., 27, 853; Olmsted, J. M. H., 370; Olson, H. L., 200; Ore, O., 24, 182, 834; Osgood, W. F., 24.

Painter, N. H., 549; Palatini, F., 372; Pall, G., 24, 523, 524; Paranjpye, R. P., 26; Parker, W. V., 175, 179; Pate, R. S., 200, 370; Pauli, W., 851; Paydon, J. F., 854; Peebles, G. H., 24; Perkins, F. W., 24; Perlis, S., 372; Pettis, B. J., 24; Phalen, H. R., 199; Pitcher, E., 179; Plummer, H. C., 548; Pólya, G., 27, 197; Poritsky, H., 24; Post, E. L., 339; Potter, M. A., 177; Prange, G., 682; Preisman, A., 850; Price, G. B., 176, 180, 834, 837, 838; Price, H. V., 370; Pringsheim, A., 855; Putnam, T. M., 179, 517; Pyle, H. R., 199.

Quade, E. S., 681.

Rademacher, H. A., 24, 834; Radó, T., 24, 180, 519, 523; Rainville, E. D., 853; Randels, W. C., 24, 27; Rappoport, A., 24; Raynor, G. E., 179; Read, C. B., 199; Reade, M. O., 370; Rees, C. J., 370, 551; Reichelderfer, P. V., 28, 854; 
Reid, W. T., 24, 182; Richardson, R. G. D., Frontispiece, 177, 180, 838, 851; Richardson, Mrs. R. G. D., 177; Rickart, C. E., 682; Rickey, F. A., 176; Rigby, F. D., 28, 370; Rinehart, R. F., 551; Ritt, J. F., 24, 834; Ritter, E. K., 854; Roberts, I. C., 200; Roberts, J. H., 24, 551; Robertson, H. P., 182, 183, 524; Robertson, M. S., 24; Robinson, C. V., 370; Robinson, G. de B., 853; Robinson, R. M., 24, 853; Room, T. G., 549; Roos, C. F., 366; Rosenbloom, P. C., 548, 549; Ross, A. E., 24; Rossby, C. G., 197; Rosser, B., 5, 24, 526; Royall, N. N., 370; Ruger, H. A., 27; Rulon, P. J., 551; Rusk, W. J., 179; Rutledge, G., 29; Rutt, N. E., 175.

Sachs, J. M., 370; Sagen, O. K., 853; Samelson, H., 851 ; Sánchez-Díaz, R., 681; Sanders, S. T., 175; Sanders, S. T., Jr., 199; Sard, A., 681; Savage, L. J., 851; Schaeffer, A. C., 173, 681; Schart, W. J., 200, 370, 853; Scheffé, H., 854; Schenberg, M., 851; Schilling, O. F. G., 24, 834; Schneckenburger, E. R., 370; Schoenberg, I. J., 24, 681; Schraut, K. C., 200, 370, 372; Schur, I., 200; Schwartz, A., 854; Schwartz, H. M., 28; Sealander, C. E., 853; Sedgewick, C. H. W., 551; Segal, I. E., 371, 372; Seidel, W., 24; Sewell, W. E., 24; Shannon, C. E., 371; Sheedy, J. W., 853; Sheffer, I. M., 24, 199, 524; Shephard, R. W., 371; Sherman, S., 371, 682; Shewhart, W. A., 334; Shobé, L. R., 200; Shohat, J. A., 24, 25, 524; Siegel, C. L., 851; Sigley, D. T., 199; Slepian, J., 549; Smail, L. L., 24; Smith, C. B., 200; Smith, J. C., 854; Smith, P. A., 182; Smith, S. S., 371; Smith, W. M., 179; Snyder, V., 329, 835; Snyder, W. S., 854; Sobczyk, A., 371; Sokolnikoff, I. S., 24, 837, 853; Specht, R. D., 682; Spitzbart, A., 371; Stafford, A. A., 853; Starcher, G. W., 551; Stark, R. H., 682; Starr, D. W., 371; Steenrod, N. E., 24, 176; Stephens, W., 177; Stevens, C. E., 681; Stevenson, A. C., 550; Stewart, B. M., 28, 371; Stewart, H. J., 173, 517; Stoker, J. J., 334; Stone, A. H., 851; Stone, M. H., 25, 182, 834, 838; Stopher, E. C., 853; Strodt, W., 200; Struik, D. J., 24; Sturley, E. A., 853; Sugar, A., 24; Sutton, C. S., 28; Szász, O., 24; Szegö, G., 24, 173, 179, 517; Szegö, Mrs. G., 517.

Tamarkin, J. D., 24, 176, 183, 334, 527; Tarski, A., 25, 680; Tate, J. L., 548; Taylor, J. H., 24, 179; Thielman, H. P., 24; Thomas, G. B., 28, 371; Thomas, J. M., 25; Thomas, T. Y., 24, 179, 549, 837; Thorne, C. J., 854; Thrall, R. M., 523, 851; Titt, E. W., 179; Topp, C. W., 372; Torrance, C. C., 551; Townes, S. B., 199; Trevor, J. E., 552; Trimble, H. C., 200; Trjitzinsky, W. J., 179; Tucker, A. W., 25, 180; Tukey, J. W., 24, 25, 551, 835, 838.

Uhler, H. S., 550; Ulam, S. M., 24; Uspensky, J. V., 24.

Van de Carr, J. C., 28; Van Schaack, G. B., 853; Van Vleck, J. H., 182, 524; Varineau, V. J., 28, 371; Varnhorn, M., 200; Veblen, O., 679; Vedova, G. C., 199; Volterra, V., 28.

Wahlin, G. E., 25; Wald, A., 366; Walker, R. J., 24, 179, 526; Wall, H. S., 5, 25; Wallace, A. D., 24, 552; Wallman, H., 199; Walsh, J. L., 24, 25, 183, 527, 838; Walton, L. F., 371; Ward, L. E., 24; Ward, M., 179, 180, 199; Watts, J. O., 372; Way, S., 334; Weaver, W., 180, 548; Webber, G. C., 199; Weber, E., 850; Webster, M. S., 24, 681; Weida, F. M., 179; Welmers, E. T., 28; Wescott, M. E., 371; de Wet, J. S., 371; Weyl, H., 180, 834; Whaples, G. W., 372, 551; Wheeler, A. P., 179; Wheeler, C. H., 682; Whitehead, G. W., 854; Whitehouse, W. W., 5; Whiteman, A. L., 371; Whitman, P. M., 552; Whitmore, W. F., 854; Whitney, B. S., 28; Whitney, H., 24; Whyburn, G. T., 180, 197; Whyburn, W. M., 837; Wiener, N., 24, 182, 524, 679, 834, 835; Wilcox, L. R., 24; Wilder, R. L., 28, 527, 834; Wilks, S. S., 182, 334, 836; Williams, K. P., 5; Williams, L. B., 200; Williams, M. H., 551; Williamson, J., 24; Wilson, G. R., 200; Wilson, R. L., 551; Win- 
bigler, A., 855; Winger, R. M., 177; Wintner, A., 24, 25; Wolf, L. A., 854; Wood, O. V., 371; Woodward, F., 832; Wray, W. D., 28; Wright, H. N., 551; Wright, S., 834; Wyman, M., 371.

Young, A., 200; Young, M. M., 550; Youngs, J. W. T., 854.

Zariski, O., 200, 527, 834, 837; Zimmerberg, H., 548, 549; Zippin, L., 24, 176; Zorn, M., 24; Zygmund, A., 197.

Prizes and Medals:

Adams Prize, 679; Charles Chree Medal and Prize, 850; De Morgan Medal, 679; Jablonowskischen Gesellschaft der Wissenschaften Prize, 851; William Lowell Putnam Mathematical Prize, 548, 680; Sylvester Medal, 198.

Universities and Technical Schools:

Beaver College, 179; Brooklyn College, 548; Brown University, 548; University of Cambridge, 679; Carnegie Institute of Technology, 549; University of Chicago, 526, 832; University of Cincinnati, 366; Cooper Union Institute of Technology, 549; Drury College, 179; Fordham University, 526; Grinnell College, 179; Harvard University, 25, 850; Institute for Advanced Study, 25, 850; The State University of Iowa, 526; Massachusetts Institute of Technology, 548; New York University, 679; Northeastern University, 179; University of Notre Dame, 366; University of Pennsylvania, 548; Rutgers University, 837; Stanford University, 173, 197; United States Naval Academy, 197; Washington University, 197; Wheaton College, 526; Yale University, 26, 549. 


\section{INDEX OF REPORTS OF MEETINGS AND MISCELLANEOUS ARTICLES}

Announcement of the Project for the Computation of Mathematical Tables, 363.

Ayres, W. L. Reports of meetings of the American Mathematical Society: November meeting in Detroit, 5; Annual meeting of the Society, 175; April meeting in Chicago, 518; Summer meeting in Chicago, 832.

Coolidge, J. L. William Caspar Graustein-In Memoriam, 343.

Hollcroft, T. R. Reports of meetings of the American Mathematical Society: October meeting in New York, 3; February meeting in New York, 333; May meeting in Washington, 523.

Morse, M. Report of the War Preparedness Committee of the American Mathematical Society and Mathematical Association of America at the Chicago meeting, 829.

Price, G. B. Mathematical Reviews offers a reading machine for microfilm, 1.

Putnam, T. M. Reports of meetings of the American Mathematical Society: November meeting in Los Angeles, 173; April meeting at Stanford University, 517.

Winger, R. M. Robert Edouard Moritz-In Memoriam, 188. 


\section{INDEX OF BOOK REVIEWS}

Agnew, R. P. See Shohat, J. A.

Albert, A. A. Introduction to Algebraic Theories. R. Baer, 849.

See Littlewood, D. E.

Amaldi, U. See Levi-Civita, T.

Baer, R. See Albert, A. A.

Baylis, C. A. See Bennett, A. A.

Bennett, A. A., and Baylis, C. A. Formal Logic. H. B. Curry, 354.

Birkhoff, G. Lattice Theory. (American Mathematical Society Colloquium Publications, vol. 25.) L. R. Wilcox, 194.

- See Rose, C. E.

Boas, R. P. See Levinson, N.

Borel, É., and Chéron, A. Théorie Mathématique du Bridge à la Portée de Tous. J. A. Greenwood, 10.

Brauer, R. See Murnaghan, F. D.

Carathéodory, C. Reelle Funktionen. I. J. W. Hurst and H. M. Schwartz, 18.

Chéron, A. See Borel, É.

Churchill, R. V. Fourier Series and Boundary Value Problems. N. Levinson, 538.

Ciocco, A. See Rashevsky, N.

Comrie, L. J., edited by. Barlow's Tables of Squares, Cubes, Square Roots, Cube Roots and Reciprocals of All Integer Numbers Up to 12,500. R. A. Johnson, 848.

Coolidge, J. L. A History of Geometrical Methods. V. Snyder, 20.

Curry, H. B. See Bennett, A. A.

Ertel, H. Elemente der Operatorenrechnung mit geophysikalischen Anwendungen. A. E. Heins, 846.

Festschrift Rudolph Fueter zur Vollendung seines sechzigsten Altersjahres, 30 VI 1940. W. W. Flexner, 22.

Flexner, W. W. See Festschrift Rudolph Fueter.

Fréchet, M. Les Probabilités Associêes à un Système d'Événements Compatibles et Dépendants. I. Événements en Nombre Fini Fixe. (Actualités Scientifiques et Industrielles, no. 859.) I. Kaplansky, 23.

Geymonat, L. See Waismann, F.

Givens, W. See Kron, G.

Gödel, K. The Consistency of the Axiom of Choice and of the Generalized ContinuumHypothesis with the Axioms of Set Theory. (Annals of Mathematics Studies, no. 3.) C. C. Torrance, 191.

Golomb, M. See Roever, W. H.

Greenwood, J. A. See Borel, E.

Grove, V. G. See Lane, E. P.

Heins, A. E. See Ertel, H.

Hille, E. See Shohat, J. A.

Hurst, J. W. See Caratheodory, C.

Ippen, A. T. See Milne-Thomson, L. M.

Johnson, R. A. See Comrie, L. J.

Jones, B. W. See Robinson, R. M.

Kaplansky, I. See Fréchet, M.

Kron, G. Tensor Analysis of Networks. W. Givens, 536.

Lane, E. P. Metric Differential Geometry of Curves and Surfaces. V. G. Grove, 192.

Levi-Civita, T., and Amaldi, U. Compendio di Meccanica Razionale. D. C. Lewis, 847. 
Levinson, N. Gap and Density Theorems. (American Mathematical Society Colloquium Publications, vol. 26.) R. P. Boas, 543.

See Churchill, R. V.

Lewis, D. C. See Levi-Civita, T.

Littlewood, D. E. The Theory of Group Characters. A. A. Albert, 357.

Lotka, A. J. Théorie Analytique des Association Biologiques. I. Principes. II. Analyse Démographique avec Application Particulière à l'Espèce Humaine. (Actualités Scientifiques et Industrielles, nos. 187 and 780.) S. S. Wilks, 532.

McCoy, N. H. See MacDuffee, C. C.

McLachlan, N. W. Complex Variable and Operational Calculus with Technical Applications. S. Saslaw, 8.

MacDuffee, C. C. Introduction to Abstract Algebra. N. H. McCoy, 539.

Milne-Thomson, L. M. Theoretical Hydrodynamics. A. T. Ippen, 352.

Murnaghan, F. D. The Theory of Group Representations. R. Brauer, 359.

Rashevsky, N. Advances and Applications of Mathematical Biology. A. Ciocco, 7.

Rider, P. R. See Treloar, A. E.

Robinson, R. M. Stencils for Solving $x^{2} \equiv a(\bmod m)$. B. W. Jones, 191.

Roever, W. H. The Weight Field of Force of the Earth. (Washington University Studies, Science and Technology, n. s., no. 1.) M. Golomb, 538.

Rose, C. E. Matrix and Tensor Algebra for Engineers and Chemists. G. Birkhoff, 847.

Saslaw, S. See McLachlan, N. W.

Schelkunoff, S. A. See Warren, A. G.

Schwartz, H. M. See Carathéodory, C.

Shohat, J. A., Hille, E., and Walsh, J. L. Bibliography on Orthogonal Polynomials. R. P. Agnew, 350.

Snyder, V. See Coolidge, J. L.; Tables.

Steenrod, N. E. See Tukey, J. W.

Tables of Sine, Cosine and Exponential Integrals. V. Snyder, 677.

Tintner, G. The Variate Difference Method. (Cowles Commission for Research in Economics Monographs, no. 5.) S. S. Wilks, 534.

Torrance, C. C. See Gödel, K.

Treloar, A. E. Elements of Statistical Reasoning. P. R. Rider, 677.

Tukey, J. W. Convergence and Uniformity in Topology. (Annals of Mathematics Studies, no. 2.) N. E. Steenrod, 353.

Valiron, G. Sur les Valeurs Exceptionnelles des Fonctions Méromorphes et de Leurs Dérivées. (Actualités Scientifiques et Industrielles, no. 570.) J. L. Walsh, 7.

Waismann, F. Introduzione el Pensiero Matematico. Translated by L. Geymonat. (Biblioteca di Cultura Scientifica, vol. 3.) A. Wedberg, 14.

Walsh, J. L. See Shohat, J. A.; Valiron, G.

Warren, A. G. Mathematics Applied to Electrical Engineering. S. A. Schelkunoff, 846.

Wedberg, A. See Waismann, F.

Wilcox, L. R. See Birkhoff, G.

Wilks, S. S. See Lotka, A. J.; Tintner, G. 


\section{INDEX OF ADDRESSES}

Curry, H. B. Some aspects of the problem of mathematical rigor, 221.

Evans, G. C. Continua of minimum capacity, 717.

Ingraham, M. H. Rational methods in matrix equations, 61.

James, H. M. Some applications of the Rayleigh-Ritz method to the theory of the structure of matter, 869.

Schaeffer, A. C. Inequalities of A. Markoff and S. Bernstein for polynomials and related functions, 565.

Sheffer, I. M. Some applications of certain polynomial classes, 885 .

Wall, H. S. Some recent developments in the theory of continued fractions, 405. 


\section{INDEX OF RESEARCH PAPERS}

Agnew, R. P., and Kac, M. Translated functions and statistical independence, 148. Albert, G. E. A note on quasi-metric spaces, 479.

Beckenbach, E. F. See Reade, M.

Bell, E. T. Note on a certain type of diophantine system, 155.

Bell, P. O. A characterization of the group of homographic transformations, 488.

- The $R_{\lambda}$-correspondent of the tangent to an arbitrary curve of a non-ruled surface, 509 .

Bergman, S. On a generalized Green's function and certain of its applications, 651.

Blanch, G. See Lowan, A. N.

Blumenthal, L. M. A new concept in distance geometry with applications to spherical subsets, 435.

Blumenthal, L. M., and Wahlin, G. E. On the spherical surface of smallest radius enclosing a bounded subset of $n$-dimensional euclidean space, 771.

Boas, R. P. A note on functions of exponential type, 750.

Brauer, A. On a property of $k$ consecutive integers, 328.

Brenner, J. The decomposition theorem for abelian groups, 116.

Cameron, R. H., and Martin, W. T. An unsymmetric Fubini theorem, 121.

Capelli, P. F. Sur le nombre complexe binaire, 585.

Chevalley, C., and Frink, O. Bicompactness of cartesian products, 612.

Chung, K.-L. Note on a theorem on quadratic residues, 514.

Clark, C. E. On the existence of electrical networks, 769.

Coburn, N. Unitary spaces with corresponding geodesics, 901.

Cohn, R. On the analogue for differential equations of the Hilbert-Netto theorem, 268. Some exceptional values of the limit of the ratio of arc to chord, 746.

Cunningham, A. B. Non-involutorial space transformations associated with a $Q_{1,2}$ congruence, 309.

Curry, H. B. See Index of Addresses.

Daum, J. A. On certain basic series, 781.

Day, M. M. Reflexive Banach spaces not isomorphic to uniformly convex spaces, 313.

- Some more uniformly convex spaces, 504.

Dines, L. L. On the mapping of quadratic forms, 494.

Dressel, F. G. A Stieltjes integral equation, 79.

Dubisch, R. Non-cyclic algebras of degree four and exponent two with pure maximal subfields, 131.

Dyer-Bennet, J. A note on finite regular rings, 784.

Eilenberg, S. An invariance theorem for subsets of $S^{n}, 73$. On spherical cycles, 432.

Eilenberg, S., and Miller, E. W. Zero-dimensional families of sets, 921.

Evans, G. C. See Index of Addresses.

Feld, J. M. Differential and integral invariants of plane curves and horn angles, 318. The geometry of whirls and whirl-motions in space, 927.

Foster, M. Note on autopolar curves, 247.

Frame, J. S. The double cosets of a finite group, 458.

Friedman, B. Fourier coefficients of bounded functions, 84 .

Frink, O. Representations of Boolean algebras, 755.

- See Chevalley, C.

Fubini, G. On the asymptotic lines of a ruled surface, 448.

Geronimus, J. The generalization of a lemma of M. S. Kakeya, 93.

Gleyzal, A. On the equation $d y / d x=f(x, y), 254$. 
Goheen, H. Proof of a theorem of Hall, 143.

Goldstine, H. H. Linear functionals and integrals in abstract spaces, 615.

Grünwald, G. Note on interpolation, 257.

- On a convergence theorem for the Lagrange interpolation polynomials, 271.

Hagen, B. L. See Rasmusen, R. B.

Hall, D. W., and Puckett, W. T. Conditions for the continuity of arc-preserving transformations, 468.

Hall, M. A problem in partitions, 804.

Hedge, L. B. Moment problem for a bounded region, 282.

Heins, M. H. A note on a theorem of Rado concerning the $(1, m)$ conformal maps of a multiply-connected region into itself, 128.

Higgins, T. J. Note on an integral of Bierens de Haan, 286.

Ingraham, M. H. See Index of Addresses.

Isaacs, R. P. The finite differences of polygenic functions, 444 .

Jackson, D. Note on certain orthogonal polynomials, 96.

Jaeger, J. C. Conduction of heat in regions bounded by planes and cylinders, 734 .

James, H. M. See Index of Addresses.

John, F. Discontinuous convex solutions of difference equations, 275.

Jones, F. B. Monotonic collections of peripherally separable connected domains, 661.

Kac, M. Note on the distribution of values of the arithmetic function $d(m), 815$.

- See Agnew, R. P.

van Kampen, E. R. Elementary proof of a theorem on Lorentz matrices, 288.

Krall, H. L. On derivatives of orthogonal polynomials. II, 261.

Krzyzański, M. Sur les solutions des équations du type parabolique dêterminées dans une région illimitée, 911.

Lehmer, D. H., and Lehmer, E. On the first case of Fermat's last theorem, 139.

Lehmer, E. See Lehmer, D. H.

Lewis, F. A. A note on the special linear homogeneous group $S L H\left(2, p^{n}\right), 629$.

Ling, D., and Recht, L. A theorem concerning the geodesics on a paraboloid of revolution, 934.

Lowan, A. N., and Blanch, G. Errors in Hayashi's table of Bessel functions for complex arguments, 291.

McCoy, N. H. Divisors of zero in matric rings, 166.

Macphail, M. S. Cesàro summability of a class of series, 483.

MacQueen, M. L. Osculating quadrics of ruled surfaces in reciprocal rectilinear congruences, 788.

Martin, M. A sequence of limit tests for the convergence of series, 452 .

Martin, W. T. See Cameron, R. H.

Mersman, W. A. Heat conduction in an infinite composite solid, 956.

Miller, E. W. See Eilenberg, S.

Montgomery, J. C. The roots of a polynomial and its derivative, 621 .

Murdoch, D. C. Note on normality in quasi-groups, 134.

Nakayama, T. A correction to " $A$ remark on the sum and the intersection of two normal ideals in an algebra," 332.

Niven, I. Sums of fourth powers of Gaussian integers, 923.

Oldenburger, R. Recurrence of symbolic elements in dynamics, 294.

Olds, C. D. On the representations, $N_{3}\left(n^{2}\right), 499$.

On the representations, $N_{7}\left(m^{2}\right), 624$.

Oppenheim, A. Rational approximations to irrationals, 602. 
Ostrowski, A. On a theorem by J. L. Walsh concerning the moduli of roots of algebraic equations, 742.

On the definition of contact transformations, 760.

Pall, G. The construction of positive ternary quadratic forms, 641.

Perlis, S. Scalar extensions of algebras with exponent equal to index, 670.

Puckett, W. T. See Hall, D. W.

Purcell, E. J. Space Cremona transformations of order $m+n-1,242$.

- Cremona involutions determined by two line congruences, 596.

Rad6, T., and Reichelderfer, P. Note on an inequality of Steiner, 102.

Rasmusen, R. B., and Hagen, B. L. Comments on canonical lines, 298.

Reade, M., and Beckenbach, E. F. An integral analogue of Laplace's equation, 633.

Mean-value surfaces, 808.

Recht, L. See Ling, D.

Reichelderfer, P. See Rado, T.

Reichenbach, H. Note on probability implication, 265.

Robinson, C. V. A characterization of the disc, 818.

Robinson, R. M. On the simultaneous approximation of two real numbers, 512.

Rosser, B. An additional criterion for the first case of Fermat's last theorem, 109.

Salem, R. On some properties of symmetrical perfect sets, 820 .

- On trigonometrical series whose coefficients do not tend to zero, 899.

Schaeffer, A. C. See Index of Addresses.

Schwartz, H. M. Sequences of Stieltjes integrals, 947.

Scott, W. T., and Wall, H. S. Value regions for continued fractions, 580.

Seidel, W., and Walsh, J. L. On approximation by euclidean and non-euclidean translations of an analytic function, 916.

Sheffer, I. M. See Index of Addresses.

Sherman, S. Some new properties of transfinite ordinals, 111.

Smiley, M. F. Measurability and modularity in the theory of lattices, 76.

- Measurability and distributivity in the theory of lattices, 604.

Sobczyk, A. Projection of the space $(m)$ on its subspace $\left(c_{0}\right), 938$.

Sugar, A. On a result of Hua for cubic polynomials, 164.

Swingle, P. M. Indecomposable connexes, 796.

Thielman, H. P. On the convex solution of a certain functional equation, 118.

Thrall, R. M. A note on a theorem by Witt, 303.

Tompkins, C. A flat Klein bottle isometrically embedded in euclidean 4-space, 508.

Tornheim, L. Linear forms in function fields, 126.

Wade, T. L. A note on subgeometries of projective geometry as the theories of tensors, 475 .

Wahlin, G. E. See Blumenthal, L. M.

Walden, E. On the mapping of the sets of 24 points of the symmetric substitution group $G_{24}$ in ordinary space upon a hyperquadric cone, 665.

Wall, H. S. See Index of Addresses.

Wall, H. S. See Scott, W. T.

Wallace, A. D. A fixed-point theorem for trees, 757.

- The acyclic elements of a Peano space, 778.

Walsh, J. L. See Seidel, W.

Webster, M.S. Maximum of certain fundamental Lagrange interpolation polynomials, 71 .

Weisner, L. Power series the roots of whose partial sums lie in a sector, 160.

White, H. S. Fourteen species of skew hexagons, 764.

Whitney, H. On regular families of curves, 145.

Wong, Y. K. On biorthogonal matrices, 424. 